\title{
Lupus nephritis flare in young patients: relapse or nonadherence to treatment?
}

This article was published in the following Dove Press journal: International Journal of Nephrology and Renovascular Disease 27 March 2014

Number of times this article has been viewed

\section{Francisco Rivera* Sara Anaya* \\ Sección de Nefrología, Hospital General Universitario de Ciudad Real, Ciudad Real, Spain}

*These authors contributed equally to this work
Correspondence: Francisco Rivera

Sección de Nefrología, Hospital General

Universitario de Ciudad Real, Spain

Tel +34629678901

Fax +34 926278000

Email friverahdez@senefro.org
Purpose: Adherence is a challenging issue in the treatment of systemic lupus erythematosus. Nonadherence has been widely addressed in patients with lupus and must be detected quickly to prevent severe complications. The cases we present illustrate the importance of adherence in young adults.

Case I: A 23-year-old Spanish woman diagnosed with severe lupus nephritis 8 years previously achieved renal remission after immunosuppressive treatment with corticosteroids and cyclophosphamide. Three years later, she developed a renal flare. Her treatment was intensified, and rituximab and mycophenolate mofetil were added. One year later, she was readmitted for a new renal flare. A blood test revealed no detectable levels of mycophenolic acid, and the patient admitted she had not taken her treatment correctly. Treatment was resumed. Four years later, the patient remains in remission.

Case 2: A 19-year-old Spanish woman was diagnosed with nephrotic syndrome due to lupus nephritis. She achieved complete remission after treatment with corticosteroids and cyclophosphamide followed by mycophenolate mofetil. Two years later, she developed a severe renal relapse that was treated with corticosteroids, cyclophosphamide, and rituximab. The response to treatment was good. Mycophenolic acid was undetectable in blood. The patient admitted that she had often missed doses before this relapse. The renal response has been maintained since she resumed her previous medications 2 years ago.

Conclusion: We conclude that the frequent and severe relapses of lupus nephritis observed in young patients may actually be due to nonadherence rather than to refractory disease. Our cases are typical examples of nonadherence that were discovered after a detailed interview with the patients and their families. We emphasize the need for clinical suspicion of nonadherence when caring for young adults with lupus.

Keywords: lupus nephritis, renal flare, nonadherence

\section{Introduction}

Lupus nephritis (LN) is one of the most severe complications of systemic lupus erythematosis (SLE). In recent years, the morbidity and mortality of LN have improved considerably owing to more appropriate use of traditional drugs, such as corticosteroids and cyclophosphamide, and the incorporation of new ones, such as mycophenolate and biologic agents. ${ }^{1}$

Since SLE is incurable, medical treatments are lifelong. The type of drug and intensity of dosing are based on clinical and analytical data. ${ }^{2}$ Adherence, defined as the extent to which patients take medications as prescribed by their health care providers, ${ }^{3}$ has proven to be a challenging issue in the treatment of SLE, and nonadherence is usually associated with poor disease control and increased mortality. Curiously, suitable 
adherence is far from achievable in many cases, especially in patients at risk for not taking their medication correctly. The specific physical and psychological characteristics of childhood and adolescence make adherence particularly difficult. ${ }^{4,5}$ Taking into account that approximately $15 \%$ of patients with SLE develop their disease during this period, detection of nonadherence is worthwhile in clinical practice. ${ }^{6}$ The enormous psychological and social impact of the disease and its treatment in young patients demand a comprehensive and multidisciplinary approach. ${ }^{7}$ However, nonadherence in LN can mimic a refractory form of the disease that confuses doctors and leads to inappropriate treatment with severe side effects.

To highlight the importance of adherence in this age group, we describe two patients with LN who did not adhere to their drug schedule. Initially, neither patient admitted to nonadherence, and the clinical course was characterized by severe renal and extrarenal flares that were treated using an intensification of immunosuppressive treatment rather than the resumption of the previous regimen.

\section{Case presentations Case I}

A 23-year-old Spanish woman was diagnosed with SLE and LN in July 2004 when she was 15 . Her disease manifested as: nephrotic syndrome (proteinuria $8 \mathrm{~g} / 24$ hours, serum albumin $1.5 \mathrm{~g} / \mathrm{dL}$ ); normal renal function (serum creatinine $0.7 \mathrm{mg} / \mathrm{dL}$ ); microhematuria; high titers of antinuclear antibodies; and presence of lupus anticoagulant. Renal biopsy revealed class V LN (membranous, the Society of Nephrology/Renal Pathology Society Classification 2003) with neither vascular nor interstitial lesions. She was treated with oral prednisone ( $1 \mathrm{mg} / \mathrm{kg} / 24$ hours) and cyclosporine A ( $200 \mathrm{mg} / 24$ hours) for 8 weeks, followed by tapering the doses of prednisone. Although partial remission was achieved, she developed several disturbing side effects, such as Cushingoid face and gingival hypertrophy, which affected adherence. She subsequently presented with symptomatic thrombocytopenia that resolved after resumption of prednisone and cyclosporine A at lower doses.

One year later, after she had achieved complete renal remission, mycophenolate mofetil (1,500 mg/24 hours) was begun to treat proteinuria ( $3 \mathrm{~g} / 24$ hours). The patient soon developed severe renal damage (relapse of nephrotic syndrome and increase in serum creatinine to $4 \mathrm{mg} / \mathrm{dL}$ ) associated with anemia and thrombocytopenia. A second renal biopsy revealed a transformation to class IV LN (diffuse proliferative glomerulonephritis) with a high activity index (Austin Index, 18/24) and a low chronicity index (Austin Index, 4/12). Therefore, she received daily pulses of 6-methylprednisolone $(500 \mathrm{mg}$ intravenous $\times 3$ ), prednisone ( $1 \mathrm{mg} / \mathrm{kg} / 24$ hours by mouth), and monthly pulses of cyclophosphamide (500 $\mathrm{mg}$ intravenous $\times 3$ ). She also required hemodialysis, owing to the presence of anasarca and poor recovery of the renal function. Given the possibility of a refractory form of LN, she received four weekly doses of rituximab $\left(375 \mathrm{mg} / \mathrm{m}^{2}\right)$. In the following weeks, renal function improved, and dialysis was stopped. Prednisone was tapered, and mycophenolate mofetil was begun (1,500 mg/24 hours). After several months with a stable clinical picture and renal function (serum creatinine $1.4-1.6 \mathrm{mg} / \mathrm{dL}$ and proteinuria 1-1.5 g/24 hours), she experienced a new worsening of renal function with nephrotic proteinuria and was readmitted for treatment. On day 5 of admission, the blood levels of mycophenolic acid, the active metabolite of mycophenolate mofetil, were undetectable $(<0.8 \mu \mathrm{g} / \mathrm{mL})$. Although the patient (and her family) initially denied poor adherence, she eventually admitted that she had not taken her treatment correctly, since she was worried about her physical appearance and other troublesome gastrointestinal side effects. After resumption of prednisone and mycophenolate, renal function improved, and proteinuria decreased to previous levels. She was discharged after starting hydroxychloroquine and sent to a psychologist to improve adherence. Four years after the event, she has not had any further flares of SLE-related LN.

\section{Case 2}

A 19-year-old Spanish woman was diagnosed with SLE in January 2008 when she was 17 . The disease manifested with pericarditis, hemolytic anemia, and high titers of antinuclear and anti-DNA antibodies. Four months later, she developed nephrotic syndrome with microhematuria and normal renal function. A renal biopsy revealed class IV LN. She was treated with daily pulses of 6-methylprednisolone $(1,000 \mathrm{mg}$ intravenous $\times 3$ ), prednisone ( $1 \mathrm{mg} / \mathrm{kg} / 24$ hours by mouth), and monthly pulses of cyclophosphamide (500 mg intravenous $\times 6$ ). She quickly achieved complete renal remission, and, after receiving the sixth pulse of cyclophosphamide, maintenance treatment was begun with mycophenolate mofetil (1,000 mg/24 hours). She subsequently complained that the mycophenolate produced gastrointestinal discomfort, so she reduced the dose on her own initiative. In fact, levels of serum mycophenolic acid were undetectable. She agreed to switch to mycophenolic acid (Myfortic ${ }^{\circledR}$, Novartis, Basel, Switzerland), which resulted in better tolerance. The renal response was complete for 2 years, after which time she was admitted with fever, polyserositis, pancytopenia, 
and increasing proteinuria. She received three intravenous pulses of 6-methylprednisolone. Given her severe leukopenia $(<200$ neutrophils $/ \mu \mathrm{L})$, she received several doses of a leukocyte-stimulating agent (filgrastim, Neupogen ${ }^{\circledR}$, Amgen Inc., Thousand Oaks, CA, USA) with a good response. After her neutrophil count recovered, pulses of cyclophosphamide were administered (six monthly doses), followed by mycophenolic acid. Three months later, she was hospitalized with abdominal vasculitis, increased proteinuria ( $2 \mathrm{~g} / 24$ hours), and impaired renal function (serum creatinine, $2.6 \mathrm{mg} / \mathrm{dL}$ ). She received a further three pulses of 6-methylprednisolone and four weekly doses of rituximab $\left(375 \mathrm{mg} / \mathrm{m}^{2}\right)$. At first, we considered the possibility of refractory $\mathrm{LN}$, and both she and her family claimed that she had taken all the medication correctly. However, after a detailed interview, the patient admitted that she had often missed taking medication because she was concerned about her physical appearance and weight gain. Indeed, she confessed to having lied as a result of family pressure and had decided to reduce her medication on her own initiative. After resuming her previous medications, starting hydroxychloroquine, and receiving psychological counseling, she was discharged and has been in clinical and analytical remission for 2 years.

\section{Discussion}

We report two representative cases of renal flare in patients with LN caused by nonadherence to treatment rather than refractory disease. These cases emphasize that nonadherence in $\mathrm{LN}$ is a challenge for clinicians, particularly in young patients for whom suitable treatment is mandatory.

Nonadherence is a major problem in the management of chronic diseases, including SLE, and should be suspected whenever a patient is receiving home oral medication and not improving as expected. Adherence of more than $80 \%$ is currently accepted as sufficient for achieving a therapeutic response in SLE. ${ }^{4}$ However, rates of nonadherence in patients with this disease vary between $10 \%-50 \%$, depending on the evaluation methods used. ${ }^{3,8}$ Despite the importance of adherence for successful medical interventions, no universally accepted measurement criteria have been established. Koneru et $\mathrm{al}^{9}$ reported that the Medication-Adherence SelfReport Inventory was a reliable measure of adherence in SLE. Indeed, very low blood hydroxychloroquine levels are an objective marker of prolonged poor adherence in patients with this disease. ${ }^{10}$ Recently developed methods include self-reported adherence ${ }^{11}$ and electronic monitoring of medication consumption. ${ }^{12}$
In the cases we report, we were alerted to poor adherence by the undetectable blood levels of mycophenolic acid, which have proven useful for monitoring activity in other settings. ${ }^{13}$ In Spain, hydroxychloroquine was not included in the standard treatment of LN until recently. Therefore, the patients described did not take this drug during their initial disease. In any case, the patients' progress would likely have been similar, because nonadherence to hydroxychloroquine could be associated with nonadherence to the remaining drugs. ${ }^{10}$ It is essential to identify patients at risk for nonadherence. ${ }^{14}$ As in many chronic diseases, nonadherence is multifactorial. Interestingly, one of the predictors of nonadherence to clinic visits - and probably to medication - was the presence of renal involvement, because patients may be unaware of active disease in the absence of significant clinical manifestations. ${ }^{15}$ Additional barriers to adherence include financial problems, fear of side effects, depression, emotional stress, psychological factors, perception of treatment as inefficacious, lack of familial support, and lower educational level, ${ }^{11,12,16-18}$ as well as young age, unmarried status, and poor communication between patients and health care providers. ${ }^{4,19}$

The two reasons for nonadherence we identified were young age and fear of adverse side effects, mainly those related to physical appearance. We report two typical examples of nonadherence that was discovered after a detailed interview with the patients and their families. Although we did not have any specific tools for detecting nonadherence, we would emphasize the importance of clinical suspicion of nonadherence when caring for young patients with SLE. Determination of the levels of mycophenolic acid in blood is also an effective tool for evaluating nonadherence, as in the present report.

Treatment of $\mathrm{LN}$ in young patients is similar to that used in adults, although it is affected by specific problems stemming from age, psychological adaptation, and exposure to side effects in the long term. ${ }^{6}$ Thus, it is not surprising that nonadherence is one of the most challenging issues when treating young patients with SLE.

First, adolescence is a time of profound biological and psychological changes, and a diagnosis of SLE with severe organ damage, such as that caused by LN, could have an impact on adaptation to this chronic disease. ${ }^{4}$ Second, many young people find drug side effects almost intolerable and are thus at risk for nonadherence, which can manifest as failure to fill prescriptions, delay in taking medication, omission of doses, failure to follow medical advice, and poor attendance at appointments. ${ }^{5}$ Third, young patients with SLE do not 
admit to their nonadherence so as not to damage their relationship with their family and health care providers. ${ }^{20}$

In the cases we describe, refractory LN was the first diagnosis, although the true cause of renal flare proved to be nonadherence. Therefore, if our patients had admitted to their nonadherence, we would have successfully administered a less aggressive treatment regimen comprising corticosteroids, cyclophosphamide, and/or mycophenolate without rituximab or repeated boluses of cyclophosphamide. Once nonadherence has been confirmed, specific interventions tailored to the patient and his/her family can be provided., ${ }^{5,16,21}$ In our patients, psychological support combined with increased communication between family, patient, and medical staff improved adherence. Several years after the renal flares, no associated problems have been reported.

\section{Conclusion}

We conclude that the frequent and severe relapses of LN observed in young patients may actually be due to nonadherence rather than to refractory disease. This possibility must always be taken into account, especially in teenagers, in whom adherence to chronic treatment is hampered mainly for psychological reasons. Prompt identification of this situation can obviate aggressive immunosuppressive treatment and facilitate psychological and familial support so that the patient can follow treatment and maintain sustained renal and systemic remission.

\section{Acknowledgment}

We thank Thomas O'Boyle for proofreading the manuscript.

\section{Author contributions}

Francisco Rivera and Sara Anaya contributed equally to the design of this work, data analysis and interpretation, and writing of the manuscript.

\section{Disclosure}

The authors report no conflicts of interest in this work.

\section{References}

1. Tesar V, Hruskova Z. Recent news in the treatment of lupus nephritis. Minerva Med. 2012;103(4):235-251.

2. Rosario C, Seguro L, Vasconcelos C, Shoenfeld Y. Is there a cure for systemic lupus erythematosus? Lupus. 2013;22(5):417-421.
3. Osterberg L, Blaschke T. Adherence to medication. $N$ Engl J Med. 2005;353(5):487-497.

4. Rapoff MA. Management of adherence and chronic rheumatic disease in children and adolescents. Best Pract Res Clin Rheumatol. 2006;20(2): 301-314.

5. Beier UH, Green C, Meyers KE. Caring for adolescent renal patients. Kidney Int. 2009;77(4):285-291.

6. Tucker LB. Controversies and advances in the management of systemic lupus erythematosus in children and adolescents. Best Pract Res Clin Rheumatol. 2002;16(3):471-480.

7. Morgan TA, Watson L, McCann LJ, Beresford MW. Children and adolescents with SLE: not just little adults. Lupus. 2013;22(12): 1309-1319.

8. Uribe AG, Alarcón GS, Sanchez ML, et al; Lumina Study Group. Systemic lupus erythematosus in three ethnic groups. XVIII. Factors predictive of poor compliance with study visits. Arthritis Rheum. 2004;51(2):258-263.

9. Koneru S, Shishov M, Ware A, et al. Effectively measuring adherence to medications for systemic lupus erythematosus in a clinical setting. Arthritis Rheum. 2007;57(6):1000-1006.

10. Costedoat-Chalumeau N, Amoura Z, Hulot JS, et al. Very low blood hydroxychloroquine concentration as an objective marker of poor adherence to treatment of systemic lupus erythematosus. Ann Rheum Dis. 2007;66(6):821-824.

11. Garcia-Gonzalez A, Richardson M, Garcia Popa-Lisseanu M, et al. Treatment adherence in patients with rheumatoid arthritis and systemic lupus erythematosus. Clin Rheumatol. 2008;27(7):883-889.

12. Marengo MF, Waimann CA, de Achaval S, et al. Measuring therapeutic adherence in systemic lupus erythematosus with electronic monitoring. Lupus. 2012;21(11):1158-1165.

13. Djabarouti S, Duffau P, Lazaro E, et al. Therapeutic drug monitoring of mycophenolate mofetil and enteric-coated mycophenolate sodium in patients with systemic lupus erythematosus. Expert Opin Pharmacother. 2010;11(5):689-699.

14. de Achaval S, Suarez-Almazor ME. Treatment adherence to diseasemodifying antirheumatic drugs in patients with rheumatoid arthritis and systemic lupus erythematosus. Int J Clin Rheumatol. 2010;5(3): 313-326.

15. Uribe AG, Ho KT, Agee B, et al. Relationship between adherence to study and clinic visits in systemic lupus erythematosus patients: data from the LUMINA cohort. Lupus. 2004;13(8):561-568.

16. Koneru S, Kocharla L, Higgins GC, et al. Adherence to medications in systemic lupus erythematosus. J Clin Rheumatol. 2008;14(4): 195-201.

17. Julian LJ, Yelin E, Yazdany J, et al. Depression, medication adherence, and service utilization in systemic lupus erythematosus. Arthritis Rheum. 2009;61(2):240-246.

18. Duvdevany I, Cohen M, Minsker-Valtzer A, Lorber M. Psychological correlates of adherence to self-care, disease activity and functioning in persons with systemic lupus erythematosus. Lupus. 2011;20(1): $14-22$.

19. Chambers SA, Rahman A, Isenberg DA. Treatment adherence and clinical outcome in systemic lupus erythematosus. Rheumatology (Oxford). 2007;46(6):895-898.

20. Bennett JK, Fuertes JN, Keitel M, Phillips R. The role of patient attachment and working alliance on patient adherence, satisfaction, and health-related quality of life in lupus treatment. Patient Educ Couns. 2011;85(1):53-59.

21. Ting TV, Kudalkar D, Nelson S, et al. Usefulness of cellular text messaging for improving adherence among adolescents and young adults with systemic lupus erythematosus. J Rheumatol. 2012;39(1):174-179. 
International Journal of Nephrology and Renovascular Disease

Dovepress

\section{Publish your work in this journal}

The International Journal of Nephrology and Renovascular Disease is an international, peer-reviewed open-access journal focusing on the pathophysiology of the kidney and vascular supply. Epidemiology, screening, diagnosis, and treatment interventions are covered as well as basic science, biochemical and immunological studies. The journal welcomes original research, clinical studies, reviews \& evaluations, expert opinion and commentary, case reports and extended reports. The manuscript management system is completely online and includes a very quick and fair peerreview system, which is all easy to use. Visit http://www.dovepress.com/ testimonials.php to read real quotes from published authors.

Submit your manuscript here: http://www.dovepress.com/international-journal-of-nephrology-and-renovascular-disease-journal 